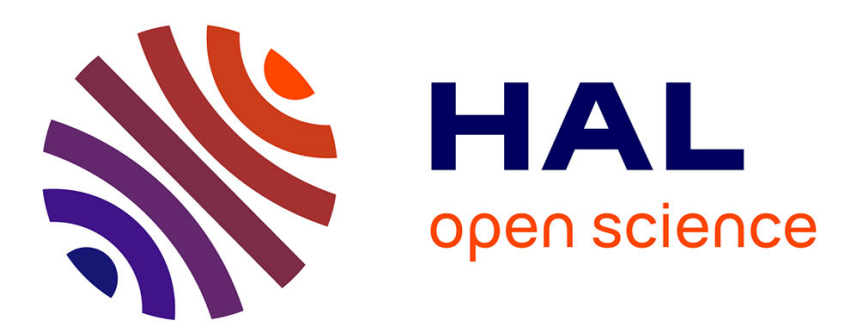

\title{
Nighttime Visibility Analysis and Estimation Method in the Presence of Dense Fog
}

Romain Gallen, Aurélien Cord, Nicolas Hautiere, Eric Dumont, Didier Aubert

\section{To cite this version:}

Romain Gallen, Aurélien Cord, Nicolas Hautiere, Eric Dumont, Didier Aubert. Nighttime Visibility Analysis and Estimation Method in the Presence of Dense Fog. IEEE Transactions on Intelligent Transportation Systems, 2015, 16 (1), pp 310-320. 10.1109/TITS.2014.2331177 . hal-01216610

\section{HAL Id: hal-01216610 https://hal.science/hal-01216610}

Submitted on 16 Oct 2015

HAL is a multi-disciplinary open access archive for the deposit and dissemination of scientific research documents, whether they are published or not. The documents may come from teaching and research institutions in France or abroad, or from public or private research centers.
L'archive ouverte pluridisciplinaire HAL, est destinée au dépôt et à la diffusion de documents scientifiques de niveau recherche, publiés ou non, émanant des établissements d'enseignement et de recherche français ou étrangers, des laboratoires publics ou privés. 


\title{
Nighttime Visibility Analysis and Estimation Method in the Presence of Dense Fog
}

\author{
Romain Gallen, Aurélien Cord, Nicolas Hautière, Member, IEEE, Éric Dumont, and Didier Aubert
}

\begin{abstract}
Compared with daytime, a larger proportion of road accidents happens during nighttime. The altered visibility for drivers partially explains this situation. It becomes worse when dense fog is present. In this paper, we first define a standard night visibility index, which allows specifying the type of fog that an advanced driver assistance system should recognize. A methodology to detect the presence of night fog and characterize its density in images grabbed by an in-vehicle camera is then proposed. The detection method relies on the visual effects of night fog. A first approach evaluates the presence of fog around a vehicle due to the detection of the backscattered veil created by the headlamps. In this aim, a correlation index is computed between the current image and a reference image where the fog density is known. It works when the vehicle is alone on a highway without external light sources. A second approach evaluates the presence of fog due to the detection of halos around light sources ahead of the vehicle. It works with oncoming traffic and public lighting. Both approaches are illustrated with actual images of fog. Their complementarity makes it possible to envision a complete night-fog detection system. If fog is detected, its characterization is achieved by fitting the different correlation indexes with an empirical model. Experimental results show the efficiency of the proposed method. The main applications for such a system are, for instance, automation or adaptation of vehicle lights, contextual speed computation, and reliability improvement for camera-based systems.
\end{abstract}

Index Terms-Advanced driver assistance systems (ADASs), advanced lighting systems, camera, fog characterization, fog detection, night fog, visibility.

Manuscript received December 10, 2013; revised March 18, 2014 and May 22, 2014; accepted June 12, 2014. This work was supported in part by Agence Nationale de la Recherche through the Dialogue Infrastructure Véhicules pour Améliorer la Sécurité Project and the Improved CAmera based Detection under Adverse Conditions (ICADAC) Project under Grant $6866 \mathrm{C} 0210$ projects.

R. Gallen is with the Information Processing Unit, Direction technique Eau, mer et fleuves (DTecEMF), Centre d'Etudes et d'Expertises sur les Risques, l'Environnement, la Mobilité et l'Aménagement (CEREMA), 29280 Plouzané, France (e-mail: romain.gallen@ developpement-durable.gouv.fr).

A. Cord is with the Laboratory on Interaction Between Vehicle Infrastructure and Driver (LIVIC), Department of Components and Systems, The French Institute of Science and Technology for Transport, Development and Networks (IFSTTAR), 78000 Versailles, France (e-mail: aurelien.cord@ifsttar.fr).

N. Hautière is with the Department of Components and Systems, The French Institute of Science and Technology for Transport, Development and Networks (IFSTTAR), 77420 Marne la Vallée, France (e-mail: nicolas.hautiere@ifsttar).

E. Dumont and D. Aubert are with the Laboratory for Road Operation, Perception, Simulations and Simulators (LEPSIS), Department of Components and Systems, The French Institute of Science and Technology for Transport, Development and Networks (IFSTTAR), 77420 Marne la Vallée, France (e-mail: eric.dumont@ifsttar.fr; didier.aubert@ifsttar.fr).

Color versions of one or more of the figures in this paper are available online at http://ieeexplore.ieee.org.

Digital Object Identifier 10.1109/TITS.2014.2331177

\section{INTRODUCTION}

$\mathbf{W}$ HILE night driving only represented $10 \%$ of total traffic, it represented $47 \%$ of fatalities in 2011 in France. Moreover, accident severity during nighttime increases by a factor of 1.7 as compared with daytime [1]. Among the factors that explain these figures, drowsiness [2], speed [3], and reduced visibility [4] are frequently cited. To address this issue, emphasis is put on the development of smarter lighting strategies for vehicles [5]. Advanced systems are being developed to automate and adjust the operation of vehicle lights to maximize visibility while preventing glare for oncoming drivers.

Among the embedded sensors that drive these systems, video cameras are most promising since they have low cost and serve different purposes. In particular, adverse weather conditions, such as rain or fog, are major concerns. First, they directly affect the safety of a driver by reducing his safety margin, i.e., low visibility distance or low friction. Second, they reduce the reliability of camera-based systems by altering image quality. For these two reasons, detecting, characterizing, and mitigating the effects of adverse weather conditions, particularly fog, is a challenge for camera-based advanced driver assistance systems (ADASs). The automatic adaptation of the intensity of lights in fog is still an open issue, although regulations allow it [6]. Fog is known for its effects on visibility, but the visual effects of fog differ between night and day [7], which explains why different camera-based detection methods are needed. Onboard daytime fog detection was pioneered in [8] and further tackled in [9] and [10]. The mitigation of daytime fog was addressed by restoring the contrast of images reduced by fog [11]. The case of heterogeneous daytime fog was also addressed in [12] and [13].

In this paper, the problem of onboard night-fog detection and characterization by camera is addressed. Contrary to daytime, the visual appearance of a road scene in fog at night strongly depends on the presence of artificial light sources in the environment. If a vehicle is alone on the road and there are no lighting fixtures, the fog is only noticeable through the backscattering of light from the headlamps. If there are oncoming vehicles or if there is a road lighting installation, the backscattered veil is no longer noticeable, but the presence of fog can be still noticed by the presence of halos around the light sources. Based on these visual effects, a night-fog detection method has been proposed in [14]. Using spectral features and a simple linear classifier, a classification of images in fog and fog-free scenes is proposed in [15]. Among the existing ADASs, some systems use the blurring of the tail lamps of a leading vehicle to determine the presence of fog, such as in 
patent JP 11-278182A. This only works when there is a vehicle ahead of the equipped vehicle. The two patented camera-based systems [16], [17] rely on the backscattered light from the headlamps in order to detect the presence of fog. They both proceed by analyzing the evolution of pixel intensities along a vertical or horizontal line in the image. Those local methods are impaired by the presence of obstacles or markings in the scene, and because the backscattered luminous level is quite low, their behavior is also impaired when there are multiple light sources in the environment, such as streetlights or headlights from oncoming traffic. For night-fog characterization, some static camera-based systems rely on the temporal comparison of images containing light sources [18]-[21]. These methods cannot be adjusted to dynamic conditions.

In this paper, we extend the night-fog detection algorithm presented in [14] by adding a novel characterization step, and we assess the method using actual video sequences and reference sensors. This method is original since it is the first method to attempt the characterization of the fog density in night driving scenarios with an onboard camera. In addition, unlike patent JP 11-278182A, the method does not require other cars or obstacles to be in front of the equipped vehicle in order to work. Unlike the patents in [16] and [17] that do not work in strongly lit environments, the system is able to deal with all sorts of lighting environments and does not use a local or photometric approach but a global image analysis process. To specify the class of the fog density that needs to be characterized by our system, we propose to use a nighttime visibility index that is based on the visibility level (VL) of a small target [22].

This paper is organized as follows. A conventional scenario to derive a standard nighttime visibility index is first proposed. The detection of the backscattered veil is then described. The detection of halos around light sources follows. Then, the fog characterization step is presented. Finally, experimental results are discussed, and perspectives for potential applications are given.

\section{Design of Conventional Scenario for Standard NightTIME VisibiLITY INDEX}

\section{A. Adrian's Model}

The ability to detect an achromatic object in a traffic situation mainly depends on four parameters, i.e., object size and luminance, background luminance, and adaptation luminance. The luminance difference required to detect an object on its background increases with the overall light level to which a driver is adapted. The luminance difference threshold was investigated by Blackwell in laboratory conditions [23]. He later proposed to use the ratio between the actual contrast and the threshold contrast as a visibility descriptor, and this socalled VL was adopted by the International Commission on Illumination (CIE) to evaluate a lighting design in terms of visual performance as follows [24]:

$$
\mathrm{VL}=\frac{C}{C_{\mathrm{th}}}=\frac{\left(L-L_{b}\right) / L_{b}}{\left(L_{\mathrm{th}}-L_{b}\right) / L_{b}}=\frac{\Delta L}{\Delta L_{\mathrm{th}}}
$$

where $C$ is the actual contrast, $C_{\text {th }}$ is the threshold contrast, $L$ is the actual object luminance, $L_{\mathrm{th}}$ is the object luminance at the threshold contrast, $L_{b}$ is the background luminance, $\Delta L$ is the actual luminance difference, and $\Delta L_{\text {th }}$ is the threshold luminance difference.

The most convenient method to calculate the threshold contrast is to use analytic functions fitted to Blackwell's laboratory data. One of the most popular among such empirical models was proposed by Adrian as follows [25]:

$$
\Delta L_{\mathrm{th}}=\left(\frac{\phi^{1 / 2}}{\alpha}+L^{1 / 2}\right)^{2} \cdot F_{p} \cdot F_{c} \cdot F_{t} \cdot F_{a}
$$

with the $\phi$ and $L$ functions depending on the background luminance given by Adrian, $\alpha$ as the angular size of the target (in minutes of arc), and $F_{p}$ depending on the detection rate needed ( 1 for $50 \%, 2.6$ for $99.9 \%$ ). $F_{c}, F_{t}$, and $F_{a}$ are correction factors applied to take into account the contrast polarity, the presentation time, and the age of the observer, respectively. Adrian provided some threshold VL values accounting for field conditions (as opposed to laboratory conditions) [26].

\section{B. VL in Nighttime Driving Conditions}

The luminance is needed to calculate the VL results from the illuminance generated by the headlamps and from the reflective properties of the illuminated surface. Because contrast determines visibility, two surfaces must be considered for the purpose of evaluating the visibility distance, i.e., that of the object to detect and that of the background. The illuminance $E$ that reaches a target at distance $d$ given a pair of headlamps, for which we know the left and right luminous intensities $I_{L}$ and $I_{R}$, respectively, emitted toward the target, is given by

$$
E=E_{L}+E_{R} \approx \frac{I_{L}+I_{R}}{d^{2}}=\frac{I}{d^{2}} .
$$

Under headlamp illumination, the relevant parameter to characterize the reflective properties of a vertical object (when its surface is not specular or retroreflective) is the diffuse reflection factor $\rho$ as

$$
L \approx \frac{\rho E}{\pi} \approx \frac{\rho}{\pi} \frac{I}{d^{2}}
$$

where $L$ is the target luminance, $E$ is the illuminance generated by the headlamps on the object, $I$ is the intensity of the headlamps in the direction of the object, and $d$ is the distance between the headlamps and the object. In headlamp visibility studies, the targets are usually considered dark, with reflection factor values between 5\% and 10\%, although values up to $25 \%$ are sometimes considered [27]. Horizontal objects (markings) and the pavement are characterized by means of the retroreflected luminance coefficient for headlight illumination $R_{L}\left(\mathrm{~cd} \cdot \mathrm{m}^{-2} \cdot \mathrm{lx}^{-1}\right)$ as

$$
L=R_{L} E_{\perp} \approx R_{L} \cdot \frac{I}{d^{2}}
$$

where $E_{\perp}$ is the illuminance generated by the headlamps on a surface perpendicular to the lighting direction at the position of 


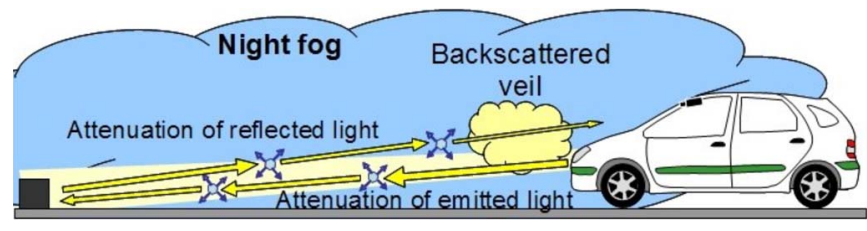

Fig. 1. Different effects of fog on light propagation in a driving scene.

the target. The $R_{L}$ of the pavement depends on the illumination and the observation geometry, but it has been shown to be independent of the distance beyond a few tens of meters, with values ranging between 5 and $30 \mathrm{mcd} \cdot \mathrm{m}^{-2} \cdot \mathrm{lx}^{-1}$ for dry pavements [28]. With the previous information, it is possible to calculate the luminance of a small target on the road illuminated by headlamps, as well as the luminance of the pavement at the base of the target, which serves for both the background luminance and the adaptation luminance [29]. For the headlamp evaluation, the exposure interval is set to $200 \mathrm{~ms}$, and a young driver is considered (age 25). These settings are used to assess the photometric visibility distance as a function of the pavement reflectivity and the headlamp beam pattern [22].

\section{Impact of Fog on Apparent Luminance}

The effects of fog on light propagation at night are illustrated in Fig. 1. Two major effects should be accounted for when the only source of illumination is the front-lighting system of the observer's vehicle, i.e., attenuation and backscattering. We estimate the attenuation of light along its path from the headlamps to the target and back to the driver using classical models of light scattering in disperse media. Then, we estimate the luminance of the backscattered veil with an empirical model based on Monte Carlo simulations.

1) Scattering of Light Depending on Distance: Fog is known for its effects on visibility. The droplets composing the fog scatter light in all directions with proportions depending on the size and number of particles per unit volume. Simpler models describe the effects of fog on perceived luminance solely based on the extinction coefficient $k$ of the Beer-Lambert exponential attenuation law, whereas more complex models take into account the distribution of droplet sizes [30]. The Beer-Lambert law describes the amount of luminous energy transmitted through a slab of fog of depth $d$ and extinction coefficient $k$ such that

$$
L=L_{0} e^{-k d}
$$

where $L_{0}$ denotes the intrinsic luminance of the considered object. Considering the visibility of a black object against the sky, the CIE has related extinction coefficient $k$ to meteorological visibility distance $V_{\text {met }}$, i.e.,

$$
V_{\text {met }}=\frac{3}{k} \text {. }
$$

Knowing the contrast threshold necessary for the detection of a target with Adrian's model and the attenuation of contrast by fog with a given extinction coefficient, we are able to compute the distance at which this minimal contrast is still perceived and, thus, the photometric visibility distance in fog. Fog induces

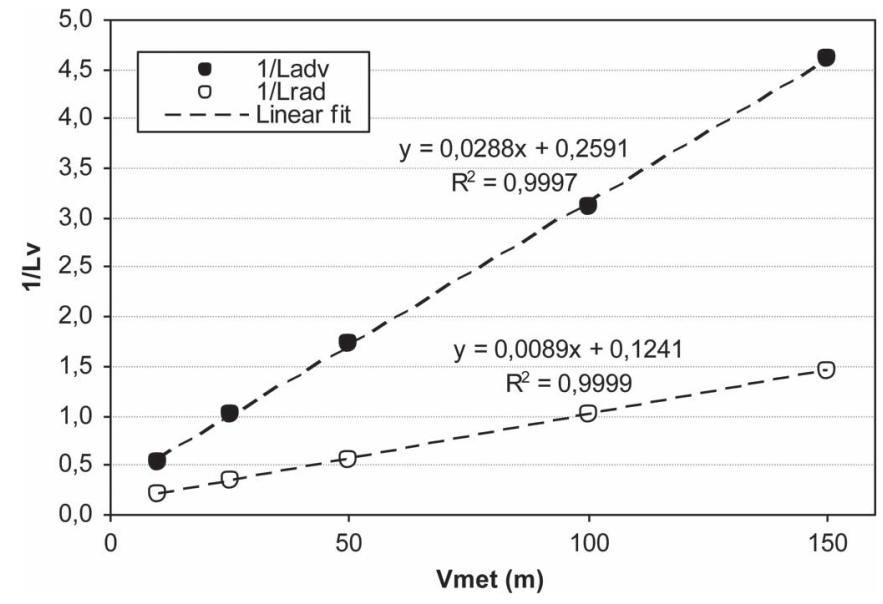

Fig. 2. Headlight backscattered veil luminance as a function of the meteorological visibility distance for two types of fog (radiation and advection).

a double attenuation of light for night driving conditions: The Beer-Lambert law first applies on the path from the headlamps to a surface in the scene and, then, back to the driver's eyes. We compose (4) and (5) with (6) in order to model this phenomenon as

$$
L \approx\left(\frac{R \cdot I}{d^{2}} e^{-k d}\right) \cdot e^{-k d}=\frac{R \cdot I}{d^{2}} e^{-2 k d}
$$

with reflectance $R$ being equal to $R_{L}$ or $\rho / \pi$, depending if we consider the pavement or the target.

2) Backscattered Veil From Headlamps: Another phenomenon that contributes to the loss of contrast in the scene is linked to backscattering. Although most of the energy is scattered forward, some is scattered backward toward the driver, generating a permanent veiling effect in front of the car. The luminance of the backscattered veil $L_{v}$ has been studied with Monte Carlo light-tracing software [31]. It has been shown to depend on both the fog type (radiation or advection [30]) and the meteorological visibility distance, and to follow this model (see Fig. 2):

$$
L_{v}=\frac{1}{a V_{\text {met }}+b} .
$$

Parameters $(a, b)$ depend on the fog type, i.e., $(0.0089$, $0.1241)$ for radiation fog and $(0.0288,0.2591)$ for advection fog. This luminance is overimposed on the driving scene, setting the driver's visual adaptation and increasing the apparent luminance of all surfaces; thus, (8) becomes

$$
L=\frac{R \cdot I}{d^{2}} e^{-2 k d}+L_{v}
$$

\section{VL Computation}

We propose to implement a photometric nighttime visibility metering tool proposed earlier [22], which we adapt for fog. The idea is to predict the VL of a small object on the roadway at any point along the road under headlight illumination based on the beam pattern, pavement retroreflectivity, and fog parameters. All geometric and photometric parameters are set conventionally: The eyes of the driver are $1.5 \mathrm{~m}$ above the 
This article has been accepted for inclusion in a future issue of this journal. Content is final as presented, with the exception of pagination.

TABLE I

Classes of Fog That Need to Be Detected by the DESIGNED IN-VeHIClE CAMERA SYSTEM

\begin{tabular}{|l||c|}
\hline Visibility range index & Meteorological visibility distance (m) \\
\hline 1 - light fog & $>300$ \\
2 - fog & 100 to 300 \\
3 - dense fog & $<100$ \\
\hline
\end{tabular}

ground, the headlamps' mounting height is $0.65 \mathrm{~m}$, the small target is a gray $0.18-\mathrm{m}$ side square with a reflection factor of $8 \%$, the headlamps' beam pattern is the average European high beam from the University of Michigan Transportation Research Institute [32], and the pavement $R_{L}$ is set between 10 and $15 \mathrm{~cd} \cdot \mathrm{m}^{-2} \cdot \mathrm{xx}^{-1}$. The VL of the target is calculated using Adrian's model, as detailed in Section II-A. Since Adrian's model cannot be inverted to compute a distance from a VL value, visibility distance $\mathbb{V}$ is iteratively obtained by setting the target closer and closer to the headlamps starting at $150 \mathrm{~m}$, until the VL reaches 7, which is the adopted threshold value.

\section{E. User Needs and Systems Requirements}

In order to define the range of the meteorological visibility distance that needs to be considered by the designed system, we define three different classes of fog.

The first class is the most critical class and happens when the nighttime visibility distance using high beams $\mathbb{V}_{\text {high }}$ is lower than the nighttime visibility distance using low beams $\mathbb{V}_{\text {low }}$ in the presence of fog. Based on our standard scenario, we are able to compute the corresponding $V_{\text {met }}$ threshold as

$$
\left.V_{\text {met }}\right|_{\mathbb{V}_{\text {high|fog }}=\mathbb{V}_{\text {low } \mid \text { fog }}} \approx 100 \mathrm{~m} \text {. }
$$

The second class happens when the nighttime visibility distance using high beams with fog becomes lower than the nighttime visibility distance using low beams without fog. Based on our standard scenario, we are able to compute the corresponding $V_{\text {met }}$ threshold as

$$
\left.V_{\text {met }}\right|_{\mathbb{V}_{\text {high|fog }}=\mathbb{V}_{\text {low } \mid \text { nofog }}} \approx 300 \mathrm{~m} \text {. }
$$

We deduce the three classes of fog, which are detailed in Table I. These classes are relevant with regard to the classes proposed in [15]. Our in-vehicle system mainly addresses fog classes 2 and 3, which correspond to the most dangerous situations.

These thresholds are critical for driver assistance and decision making in terms of lighting automation. They link physical quantities $\left(V_{\text {met }}\right)$ to practical user needs. For instance, below $V_{\text {met }}=100 \mathrm{~m}$, it is recommended to switch from high beams to low beams, bearing in mind that the visibility is much reduced as compared with clear weather. Between 100 and $300 \mathrm{~m}$, high beams are still preferable, but the visibility distance is inferior to that in clear weather with low beams. When $V_{\text {met }}$ is above $300 \mathrm{~m}$, high beams are preferred, although their range is lowered as compared with clear weather.

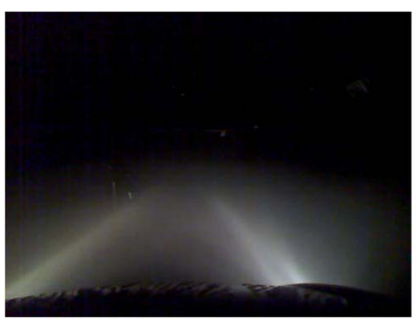

(a)

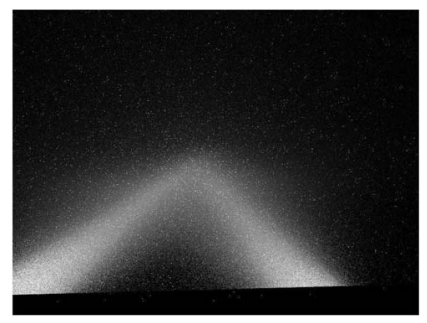

(b)
Fig. 3. Backscattered veil. (a) Actual image grabbed in the Clermont-Ferrand fog chamber [33]. (b) Simulated image using semi-Monte Carlo ray-tracing software [31].

\section{Detection of Fog And Estimation of METEOROLOGICAL VisibILITy INDEX}

Based on two visual effects of night fog, a system composed of two detection algorithms has been developed. The first algorithm detects the presence of a backscattered veil. The second algorithm detects the presence of halos around artificial light sources in the environment.

\section{A. Detection of Backscattered Veil}

The first system is based on the fact that the light emitted from the headlamps of a vehicle is scattered backward toward the driver in the presence of fog. The idea that is investigated to detect it is to compare different reference images with the input images grabbed by the camera using a correlation index.

The reference image represents the front of the vehicle with lighting on, as can be seen in fog on a dark road. It is either a real image taken with the same camera in real or artificial fog [see Fig. 3(a)], or a synthetic image. The process allowing to generate such an image can be of any type. In our case, it relies on a Monte Carlo method allowing the synthesis of images in fog in 3-D scenes [see Fig. 3(b)] [31]. When generating a reference image, the photometry and position of the headlamps, the position of the camera inside the car (including its relative angle as compared with the axis of the car), the focal length, and the image resolution of the camera should be all known in order to generate an image as close as possible to a real image acquired with the camera. The synthetic images are luminance maps. Conversion into 8-bit images is done by normalizing the luminance data between 0 and 255. A $\gamma$ function is also applied to the synthetic images in order to match the $\gamma$ of the camera. Six reference images are produced in this way for different meteorological visibility distances (see samples in Fig. 4).

Due to the temporal stability of the backscattered veil with time, the input image is a composition of $n$ successive images grabbed by the in-vehicle camera. The composition is computed by taking the mean of successive pixel values or by applying a rank filter such as the median (see Fig. 5). As one can see, the backscattered veil remains in the image, whereas other objects such as lane markings mostly disappear.

The input image is then compared with the six reference images using an image correlation index. Since the exposure settings of the camera are unknown, the synthetic and real images have different dynamics. Among available correlation distances, zero-mean normalized cross correlation (ZNCC) has 

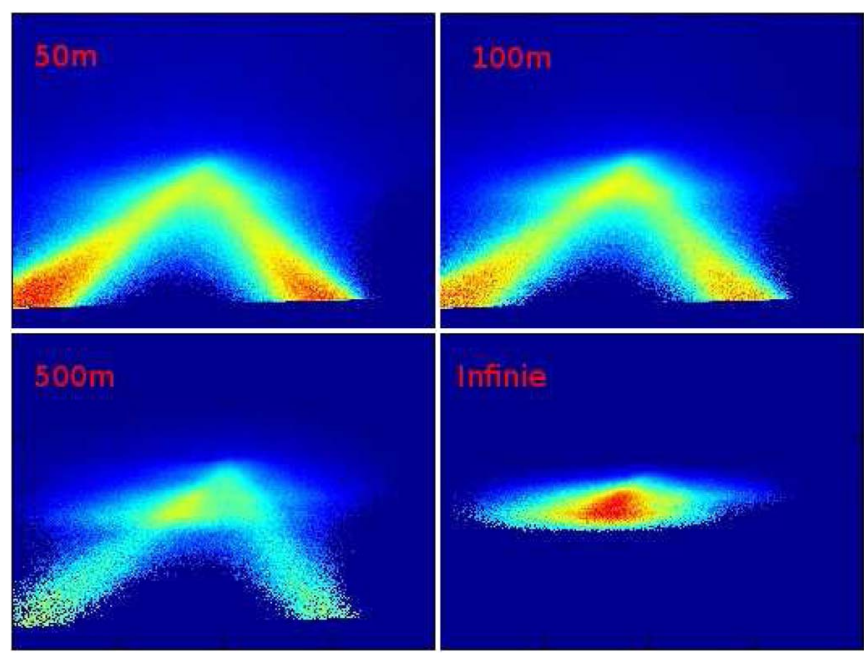

Fig. 4. Database of simulated images with different meteorological visibility distances. Color scale goes from blue to red in order to bring out luminance variations. From these luminance maps, a mask matching the shape of the backscattered veil is extracted.

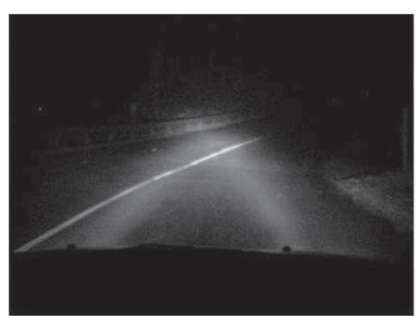

(a)

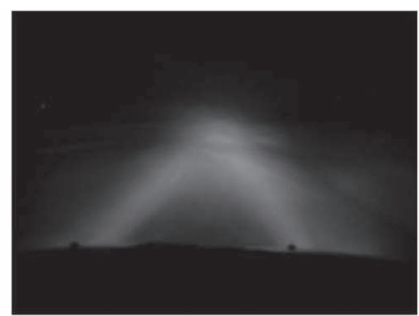

(b)
Fig. 5. Video sequence grabbed in actual fog during a test drive. (a) Sample frame. (b) Mean of successive frames. The objects in the scene are suppressed, whereas the backscattered veil remains due to its temporal stability.

invariant properties regarding the mean and scale of the illumination level. For these reasons the ZNCC has been used. Since both images are dark outside of the backscattering veil, it adds a bias in the ZNCC score and decreases its discrimination power (see Fig. 4). To overcome this problem, a mask image matching the shape of the backscattered veil in the reference image is computed using Otsu's binarization method [34]. The ZNCC score is only computed inside this mask. By applying a threshold on the correlation score, the presence of fog is assessed. When using the six reference images, six ZNCC scores with the input image are obtained; the highest score among them is selected for fog detection.

\section{B. Detection of Halos Around Light Sources}

When light sources appear in the road environment, the backscattered veil is no longer noticeable because of the limited dynamic range of the camera. However, fog induces the presence of halos around light sources. A halo is the consequence of light scattering produced by water droplets. In the image, it appears as a luminous shape around light sources, the intensity of which slowly decreases away from the source center. Its expanse and its intensity decrease mainly depend on the fog density and droplet sizes. The algorithm aiming at detecting and characterizing halos is decomposed into the following steps.
1) Light source detection. It is based on an image thresholding at a high intensity level, which is close to the maximum value of image pixels. Each connected component is extracted, and several geometrical parameters are evaluated, i.e., the position of the center of gravity $(\mathrm{CoG})$, the surface, the compactness, the elongation. Relying on these parameters, connected components that do not correspond to isolated sources are discarded. For instance, lane markings are excluded based on their shape; some sources that are agglomerated due to their proximity in the image are discarded based on their large surface or their complex shape. The remaining connected components are called "potential sources." As illustrated in Fig. 6, we start building a tree, in which the leaves are the potential sources associated with their geometrical parameters.

2) Halo segmentation. Progressively decreasing the threshold results in the connected components encompassing larger parts of each source halo in addition to the source itself. At each threshold decrement, the connected components are extracted using the potential sources as seeds. A new node is added to the tree, under the corresponding potential source's leaf, containing the same geometrical parameters. When two or more potential sources belong to the same connected component, their branches are merged into a single node. We continue decrementing the threshold until all the potential sources merge. Notice that we do not add new leaves to the tree during this process.

3) Halo selection. Based on the geometrical parameters calculated at each step, we exclude the branches for which the following are true.

a) The size growth between successive decrements is large, typically more than five times; it corresponds to the fusion of the potential source with a bright object present in the scene.

b) The gravity center drastically evolves between successive decrements without a preferred direction; it generally does not correspond to light sources.

c) Determination of halo direction. Each branch of the tree is independently studied, and the CoG of the segmented shapes is computed for each node. A linear regression on the successive positions of the $\mathrm{CoG}$ along a branch gives the direction of the halo. When a source is isotropic (all CoGs are merged), a direction is chosen arbitrarily.

d) Halo intensity profile extraction. The intensity profile is extracted along the direction estimated in the previous step, beginning at the $\mathrm{CoG}$ of the source segmented during Step 1 (Light source detection). Fig. 7 illustrates how crucial the direction choice is: For directional light sources, the halo is only present in the lighting direction (the green direction in this case).

e) Characterization of the intensity profile of the halo. In the presence of fog, the decrease in intensity along a source direction is smoother than in the absence of fog. This feature enables to detect fog presence. The choice of the curve characteristic is crucial. A large set of characteristics was explored and statistically 


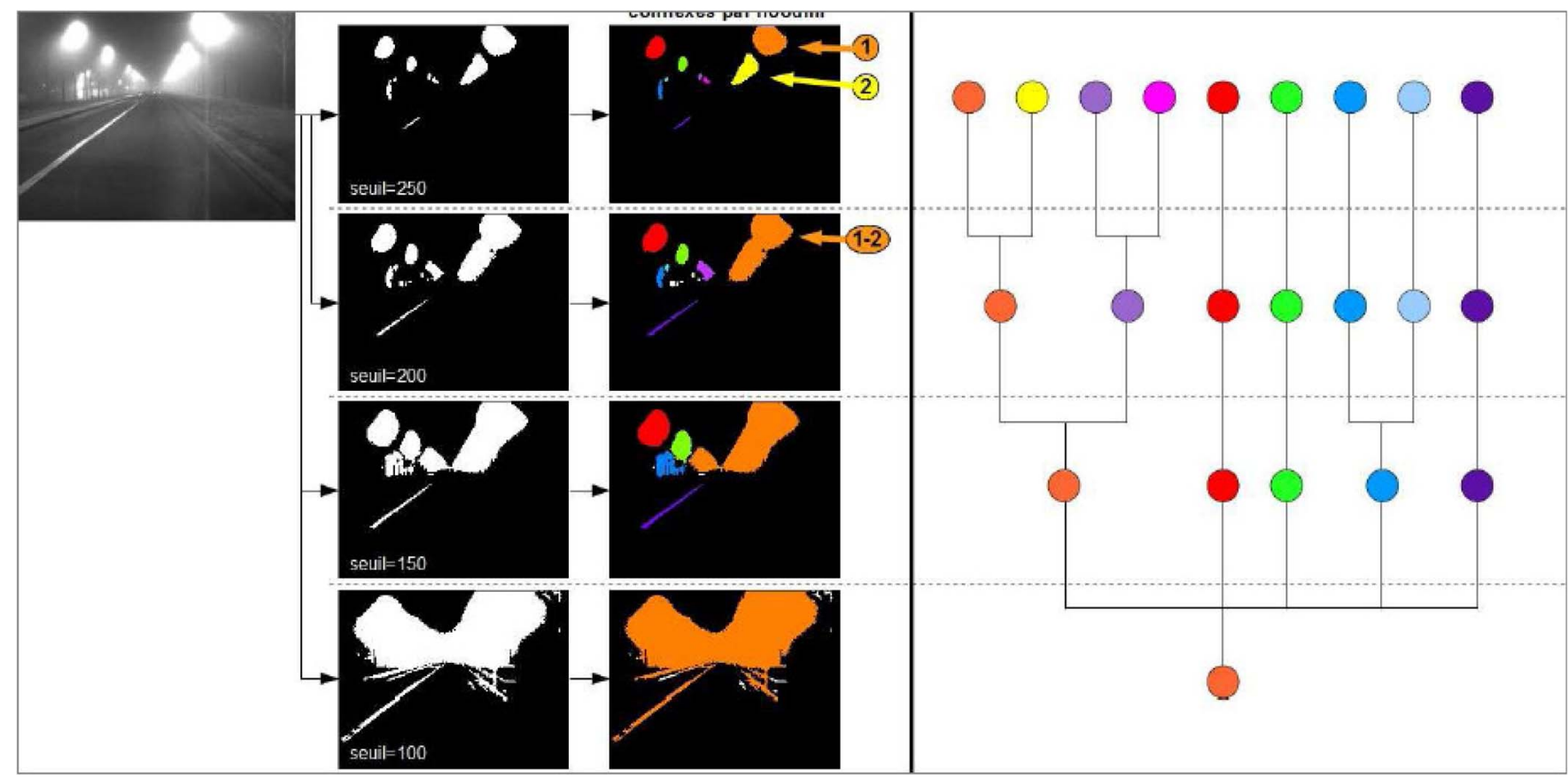

Fig. 6. Detection of light sources and associated halos. (Left) Original image. (Middle) Segmented images with decreasing thresholds and associated connected components. (Right) Tree of potential sources built using the segmented images.
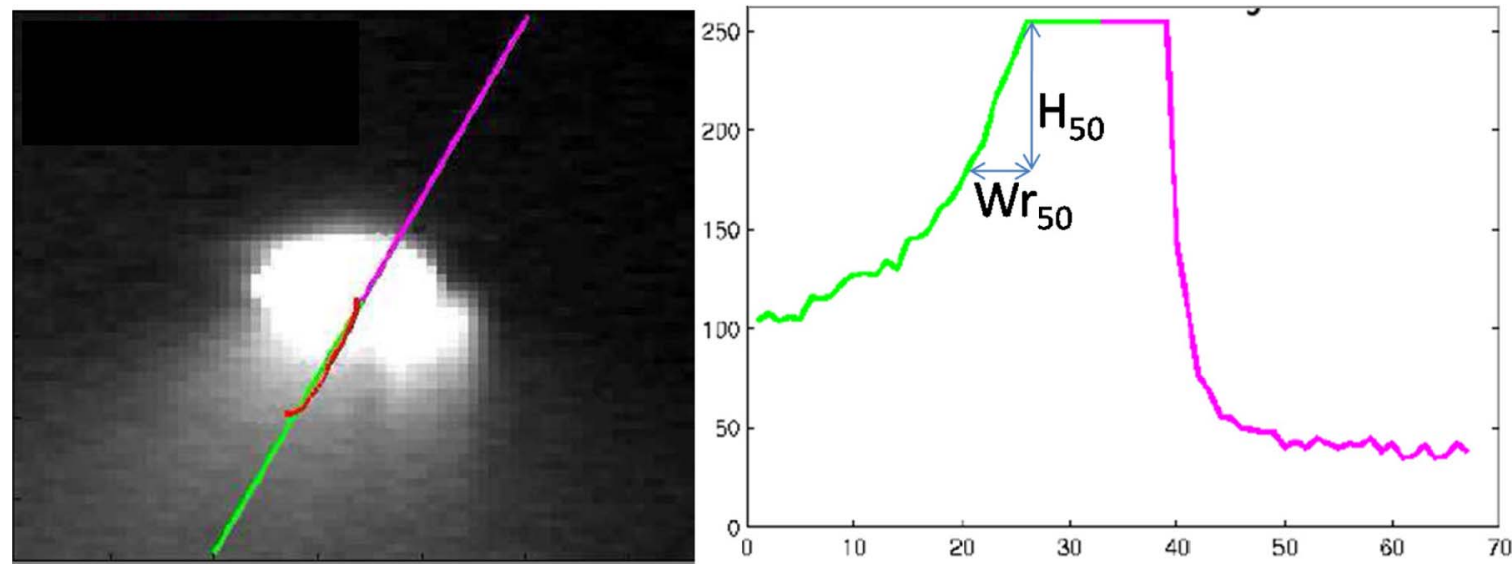

Fig. 7. Intensity profile across the halo of a directional light source. The successive positions of the CoG (in red) and the linear regression in the direction of lighting (in green) and in the opposite direction (in purple).

compared in order to select the most relevant characteristic for distinguishing between the presence and absence of fog. As illustrated in Fig. 7, the following curve characteristic is chosen: $B r_{50}=H_{50} / W r_{50}$, with $H_{50}$ as half the height of the decrease, and $W r_{50}$ as the width between the end of the sensor saturation and the curve point corresponding to the half of the height. $B r_{50}$ decreases in the presence of fog.

f) $\mathrm{Br}_{50}$ combination. Each frame of a video sequence contains several potential light sources. For each frame, the $B r_{50}$ characteristics of the different halos are averaged to compute their mean value $\overline{B r}_{50}$.

Finally, if $\overline{B r}_{50}$ is smaller than a given threshold, fog is detected. Otherwise, no fog is detected. The optimal threshold on $\overline{B r}_{50}$ in our database was estimated to be 9 . The detection process is illustrated in Fig. 8.

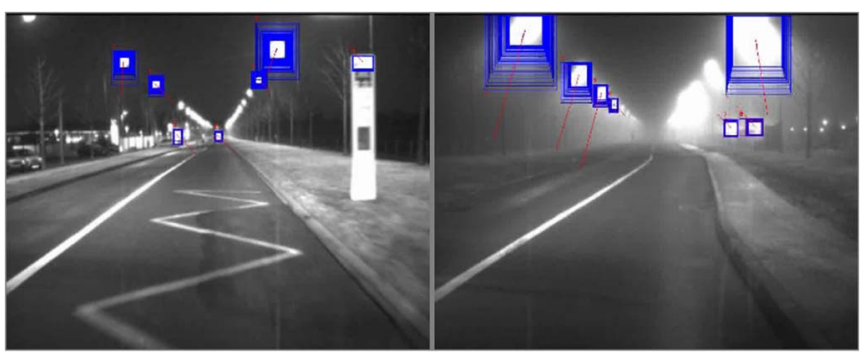

Fig. 8. Illustration of source halos' detection. (Left) Without fog. (Right) With fog. Expanding blue boxes represent the bounding boxes of the connected components at each step.

\section{Combination of Both Approaches}

As detailed previously, fog creates two main visual effects during nighttime. First, the headlamps produce a backscattered veil in the driver's field of view. Second, the external light 


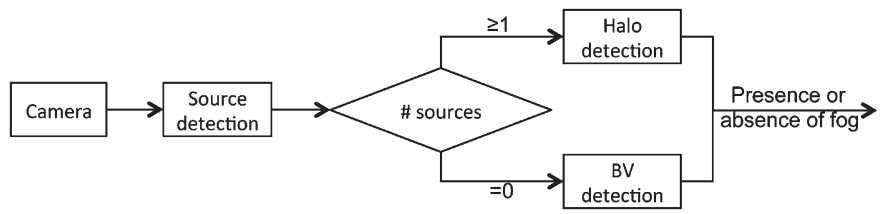

Fig. 9. Scheme of the method for combining both algorithms. The light source detection algorithm triggers the halo detection algorithm and, ultimately, the backscattered veil detection algorithm.

sources, such as public lighting or other vehicles' lights, generate a halo effect. Moreover, the backscattered veil becomes invisible when external light sources illuminate the scene, which is due to automatic camera settings and the reduced dynamic range of the camera. Two detection algorithms were presented in the previous section. The first algorithm aims at detecting the presence of a backscattered veil. The second algorithm aims at detecting the presence of halos around artificial light sources in the environment. Their combination is achieved by running them alternatively. Halo detection is run first. It starts with the detection of light sources in the field of view. If at least one source is detected, the halo detection algorithm carries on to evaluate the presence of fog based on the shape of the halo around the source(s). If no source is detected, the halo detection algorithm is interrupted, and a signal is sent to the backscattered veil detection algorithm. Without any external light source in the environment, the veil is detectable, and the correlation with reference images is relevant for evaluating the fog presence. Fig. 9 presents a scheme that summarizes this approach.

\section{Fog Characterization Through Backscattered Veil}

Both the halo and backscattered veil detection algorithms may be used to characterize the fog density and to estimate the meteorological visibility. Since the halos around light sources depend on the distance from the camera (which is unknown) and on the type and density of fog, $\overline{B r}_{50}$ does not suffice to accurately characterize fog and, thus, to estimate the meteorological visibility: It is an ill-posed problem. On the other hand, the algorithm to detect fog through the backscattered veil is based on the comparison between the current camera image and a set of reference images, which were produced under different fog densities. The correlation score serves to evaluate the fog presence. We propose to implement it to characterize the fog density. The initial fog characterization comes from the reference image with the strongest correlation score. The fog density corresponding to this reference image gives a first approximation of the actual fog density. However, this characterization is quite unstable, particularly when the density is between two reference images. It does not take into account all available information. When using several reference images, we get several comparison scores with the initial image. Combining all the scores produces a more accurate estimate of the observed fog density. For the combination, we fit the curve of the correlation score $S_{c}$ versus meteorological visibility distance $V_{\text {met }}$ with the following function:

$$
S_{c}=e^{a V_{\mathrm{met}}^{2}+b V_{\mathrm{met}}+c}
$$

where $a, b$, and $c$ are the fitting parameters. Relying on the previous equation, it is possible to estimate the current visibility distance using

$$
\tilde{V}_{\text {met }}=\frac{-b}{2 a} .
$$

Since (14) does not depend on the $c$ fitting parameter, the proposed method allows detecting and characterizing the fog density without any hard threshold on the ZNCC score. Indeed, the ZNCC score depends on the type of input image and reference image; thus, we consider its variation instead of its actual value.

\section{EXPERIMENTAL EVALUATION}

\section{A. Materials}

Weather centers are unable to forecast fog presence, which makes it very difficult to obtain experimental data. Nevertheless, different kinds of materials have been progressively collected between 2008 and 2012 to assess the performance of the presented methods. For qualitative evaluation, different short videos were acquired with an experimental vehicle in the area of Versailles (France) during successive winters; in one of these videos, a prototype LIDAR was used to provide a rough estimate of the meteorological visibility distance. For quantitative evaluation, two video sequences were produced in the fog chamber of Clermont-Ferrand (France), which is equipped with reference transmissometers [33]; however, due to fog inhomogeneity inside the chamber, these measurements cannot be considered absolute ground truth data. The whole process from fog detection to fog characterization is performed in real time using a video of resolution $1280 \times 800$ at $10 \mathrm{frames} / \mathrm{s}$. The hardware used for the experiments is an Intel Core i7 at $3 \mathrm{GHz}$. The program runs on the Intempora RT-Maps platform and is compiled with the Microsoft Visual C++ 2005 framework.

\section{B. Night-Fog Detection}

First, we illustrate the results obtained using the halo detection algorithm. We remind that a value $\overline{B r}_{50}$ lower than 9 corresponds to the presence of fog. To assess the ability of the halo detection algorithm to detect foggy weather, four video sequences were produced using an embedded camera on the same location at night, i.e., two of them during clear nights and the other two during foggy nights. They present 2130 images that contain an average of ten light sources per image. Fig. 8 illustrates the source and halo detection process on two images extracted from the database. Considering each frame independently, the proposed algorithm produced a fog detection rate of $98.4 \%$. By producing a sliding average of the characteristic of the halo profile on $1 \mathrm{~s}$ of video (25 images), the fog detection rate reached $99.86 \%$. In Fig. 10, the halo detection algorithm is further illustrated on other different driving scenarios. In the top-left image, a single source is detected. The propagation direction, which is plotted in green, corresponds to the direction of the halo. A value smaller than 9 indicates the presence of fog. 


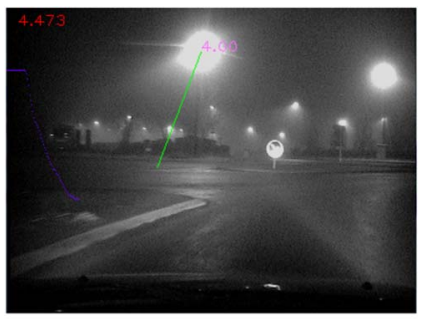

(a)

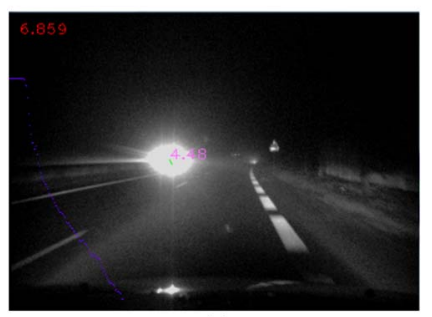

(c)

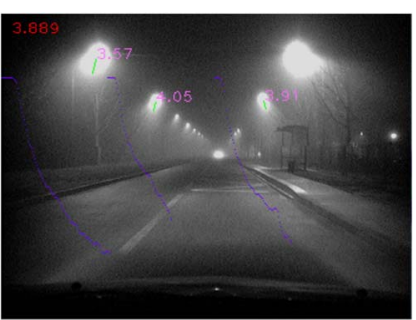

(b)

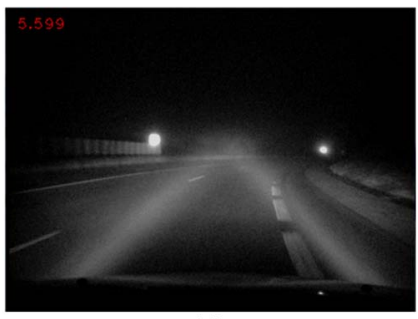

(d)
Fig. 10. Illustration of the results obtained using the halo detection algorithm. In each image, the value of the $\overline{B r}_{50}$ criterion averaged over 50 sources is presented in red in the top-left corner. A value smaller than 9 corresponds to the presence of fog. For each detected source, the halo direction is plotted in green, the $\overline{B r}_{50}$ criterion is written in pink, and the intensity profile is plotted in violet. (a) $\overline{B r}_{50}=4.473$. (b) $\overline{B r}_{50}=3.889$. (c) $\overline{B r}_{50}=6.859$. (d) $\overline{B r}_{50}=5.599$.

Notice that the road sign, which could have been detected as a source, is discarded due to the source filtering process. In the top-right image, the detection of multiple sources is illustrated. The propagation directions are different for each source, but the $\overline{B r}_{50}$ values are close $(3.57,3.91$, and 4.05$)$. In the bottomleft image, the headlamps of an oncoming vehicle are detected. The lane separation disturbs the detection, but the $\overline{B r}_{50}$ value is stable enough and is relevant to confirm the presence of fog whenever detection is possible. In the bottom-right image, no external light source is detected, but the backscattered veil is strongly present. To illustrate the halo detection algorithm, a video of the same street with and without fog is attached in this paper.

To validate the backscattered veil algorithm, we have used a recorded video in the Clermont-Ferrand (France) fog chamber. Sample results are given in Fig. 11. A high correlation score (greater than 0.6) is obtained when the measured and reference images correspond to dense fog (see Table I). In the presence of fog, this score becomes smaller (around 0.4). In the presence of light fog, it becomes very small (less than 0.2). Consequently, the confidence in fog detection based on the backscattered veil increased with the fog density. In Fig. 12, the results of the backscattered veil algorithm are presented on openroad fog images. The signal-to-noise ratio is strongly increased by averaging successive frames. The highest correlation score corresponds to the reference image having a visibility of $500 \mathrm{~m}$, which is quite consistent with the output of the dedicated LIDAR of $368 \mathrm{~m}$. However, using all correlation scores will improve the fog characterization.

\section{Night-Fog Characterization}

To validate the night-fog characterization process, we have used a video from the Clermont-Ferrand (France) fog chamber

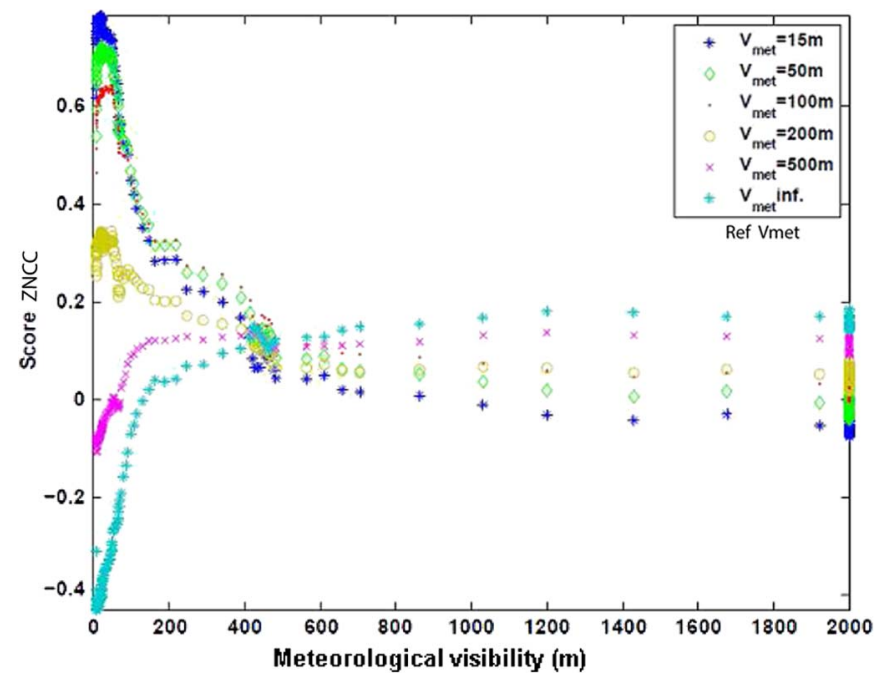

Fig. 11. ZNCC correlation score of different reference images with fog images grabbed as fog dissipates in the Clermont-Ferrand fog chamber.

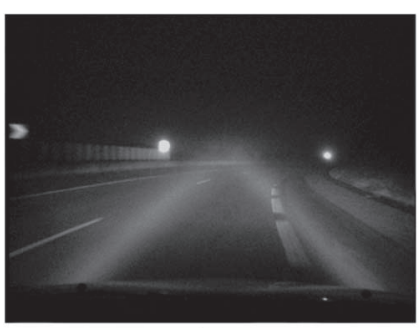

(a)

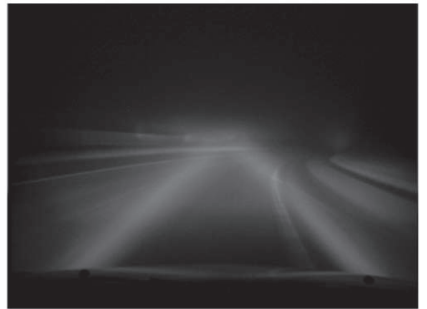

(b)

\begin{tabular}{|c||c|}
\hline Ref Vmet & ZNCC \\
\hline $15 \mathrm{~m}$ & -0.32 \\
$50 \mathrm{~m}$ & 0.075 \\
$100 \mathrm{~m}$ & 0.32 \\
$200 \mathrm{~m}$ & 0.46 \\
$500 \mathrm{~m}$ & 0.57 \\
$\infty$ & 0.42 \\
\hline
\end{tabular}

Fig. 12. Illustration of results obtained using the backscattered veil detection algorithm. (Top left) Current image. (Top right) Mean of successive images (the background image). (Bottom) Value of the ZNCC correlation scores between the background image and the six simulated reference images (15, 50, 100, 200, $500 \mathrm{~m}$, and $\infty$ ). The meteorological visibility distance measured by the dedicated LIDAR is $368 \mathrm{~m}$.

[33]. First, we have selected six frames as reference images; they correspond to the following measured visibility distances: 12.6, 51.4, 91.8, 167.8, 415.6, and $2000 \mathrm{~m}$. Then, we have computed the correlation of the 50 frames sampled from the video with the six reference frames; for each of these test frames, we have used the six correlation scores to fit the curve based on (13), and we have used that curve to estimate the meteorological visibility distance. The results for three test frames are presented in Fig. 13 with the fitted curve in green. For comparison purposes, we have also computed the correlation scores of these three test frames with the other 50 frames sampled from the video; the resulting curves are plotted in blue in Fig. 13. The similarity between both curves is satisfactory, meaning that the choice of the model and of the six reference frames to fit the curve is adapted to the problem while limiting the computing load. Moreover, as shown in the title of each graph in Fig. 13, the estimated visibility is rather close to the reference visibility. Finally, the estimated visibility distance is 


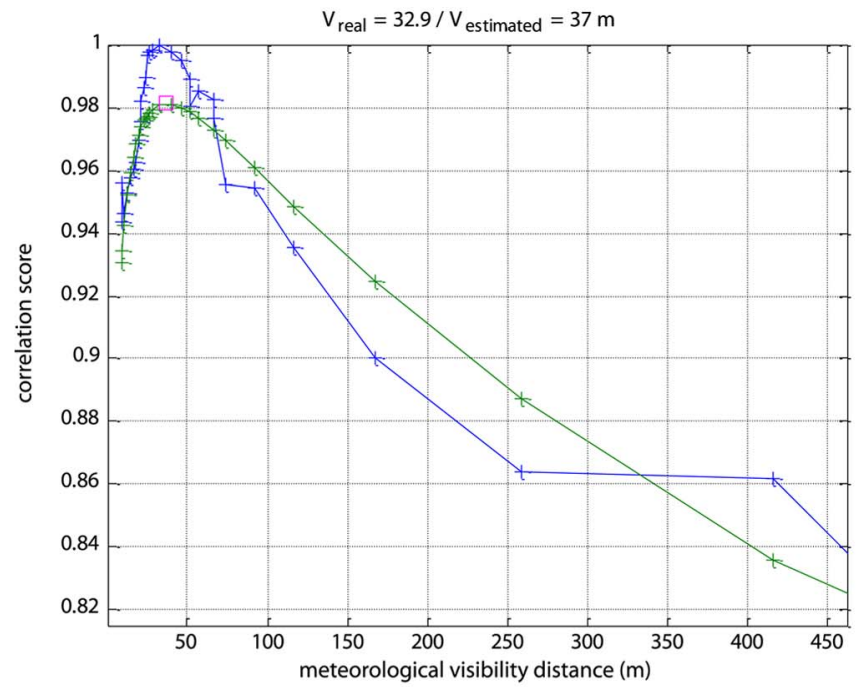

(a)

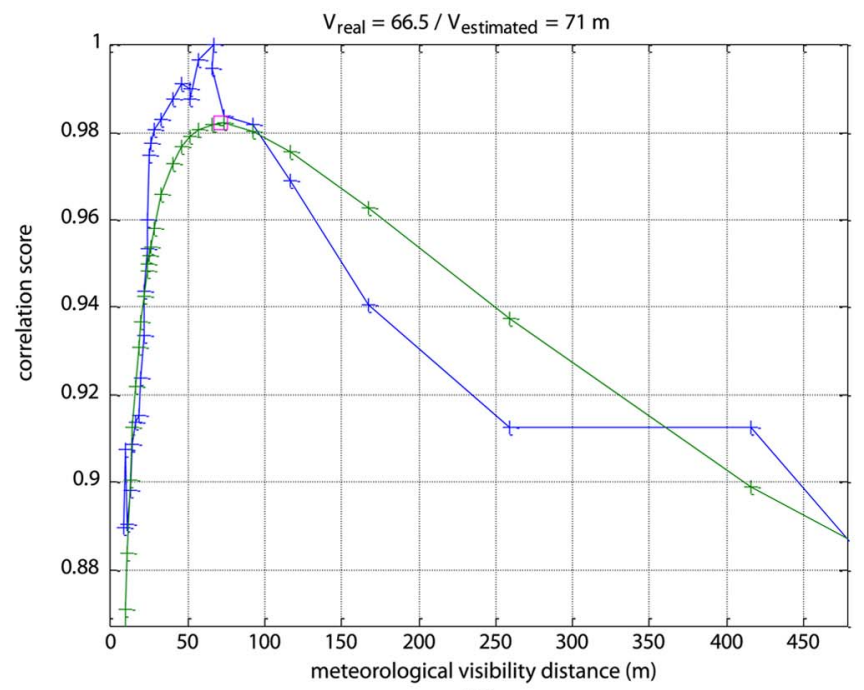

(b)

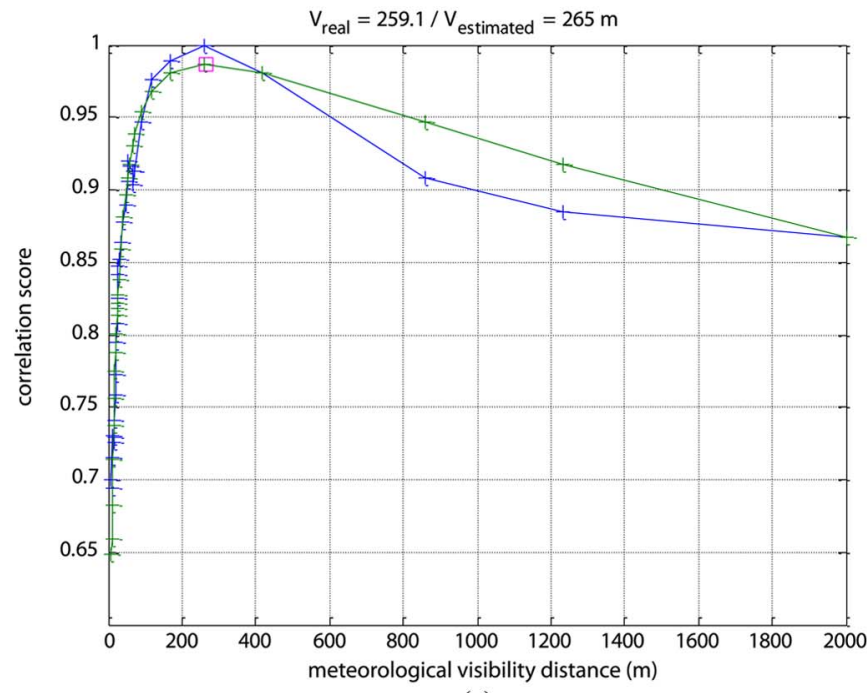

(c)

Fig. 13. Correlation scores versus the visibility distance for three different frames (a), (b), and (c) of the video acquired in the fog chamber of ClermontFerrand. The plot of the correlation score between each test frame and the 50 other frames is in blue. The fitting of the curve only using six points of the curve (reference frames) is in green.

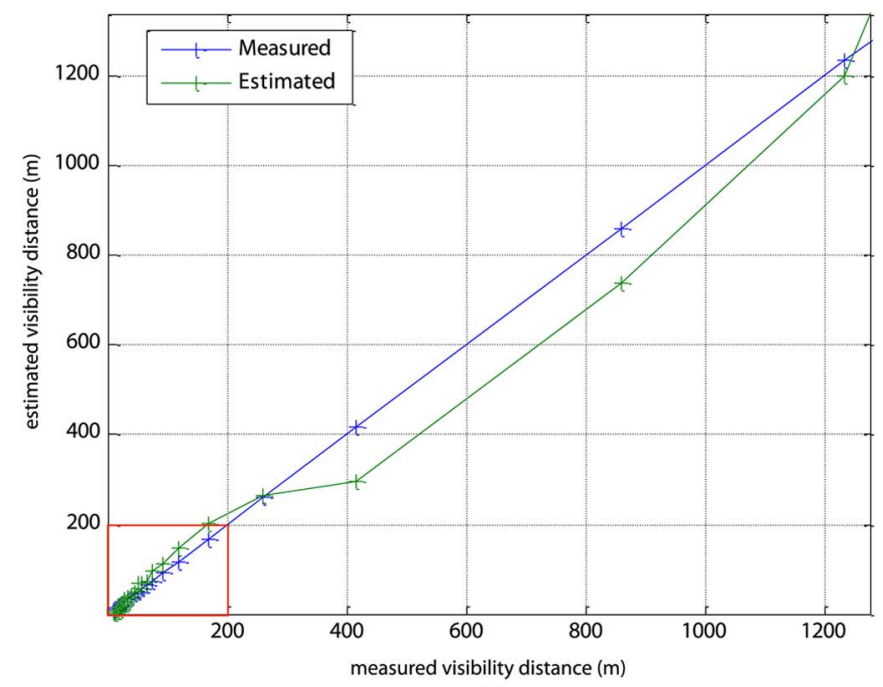

(a)

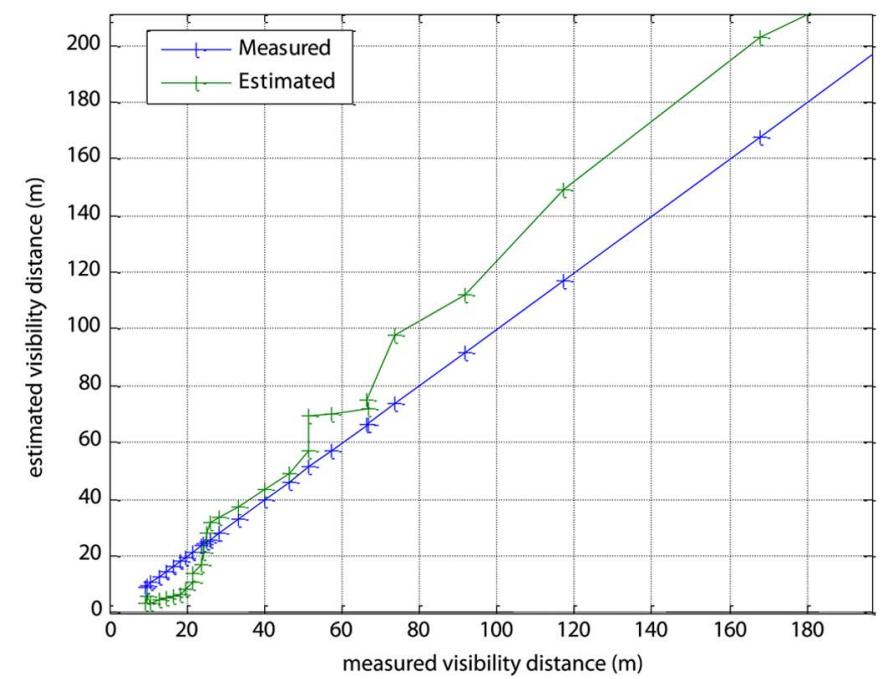

(b)

Fig. 14. Measured and estimated visibility distances obtained on a video sequence acquired in the fog chamber of Clermont-Ferrand. (a) Curve for the whole range of meteorological distances. (b) Zoom of the red square for the low meteorological visibility distances.

presented in Fig. 14 as a function of the reference visibility distance for each frame sampled from the video. The accuracy of the estimation is as follows:

- about $10 \mathrm{~m}$ of accuracy when $V_{\text {met }}<50 \mathrm{~m}$;

- about $20 \mathrm{~m}$ of accuracy when $V_{\text {met }}<100 \mathrm{~m}$;

- about $40 \mathrm{~m}$ of accuracy when $V_{\text {met }}<200 \mathrm{~m}$;

- Less than $100 \mathrm{~m}$ of accuracy when $V_{\text {met }}>200 \mathrm{~m}$.

Notice that this method is clearly better than choosing the best correlation score to infer the visibility distance. However, the accuracy is not high enough to be used directly. Nevertheless, according to these results, the designed process meets the requirements expressed in Section II-E and is able to classify the fog density in the proposed categories.

\section{Conclusion and Perspectives}

In this paper, the problem of night visibility in the presence of fog has been tackled. An original approach allows us to propose a standard night visibility index inspired by literature 
on public lighting. Based on this index, three classes of fog density directly related to user needs are presented, two of which concern the safety of drivers and should be required in the safety systems that will equip vehicles in the future. To this aim, we propose a methodology to detect the presence of night fog and to estimate the resulting meteorological visibility distance. The detection is carried out by detecting the presence of a backscattered veil or halos around external light sources. The characterization is obtained by fitting an empirical model with the correlation scores computed between the current image and the reference images with known meteorological visibility distance. The experimental results, which are both qualitative and quantitative, demonstrate the capacity of the proposed method to meet the proposed requirements in terms of accuracy. In the future, night-fog characterization will be done using halo characteristics. In this aim, we plan to collect a larger database of fog images with the associated meteorological visibility. The proposed methodology has been patented [35] and will potentially allow the development of different applications. First, it enables novel intelligent speed adaptation strategies, which are able to take into account a reduction of the visibility distance, e.g., see [3]. Second, it allows developing new adaptation strategies for vehicle front-lighting and signaling systems [36]. Finally, such a method may be used to detect and characterize fog from a fixed roadway camera [37].

\section{REFERENCES}

[1] "Statistiques d'accidentalité Routière: Résultats Définitifs de l'année 2011,” Paris, France, Tech. Rep., Jan. 2012.

[2] F. Friedrichs and B. Yang, "Camera-based drowsiness reference for driver state classification under real driving conditions," in Proc. IEEE Intell. Veh. Symp., San Diego, CA, USA, Jun. 2010, pp. 101-106.

[3] R. Gallen, N. Hautière, A. Cord, and S. Glaser, "Supporting drivers in keeping safe speed in adverse weather conditions by mitigating the risk level," IEEE Trans. Intell. Transp. Syst., pp. 1558-1571, Dec. 2013.

[4] S. Plainis, I. Murray, and I. Pallikaris, "Road traffic casualties: Understanding the night-time death toll," Inj. Prev., vol. 12, no. 2, pp. 125-128, Apr. 2006.

[5] M. Tideman and S. J. Janssen, "A simulation environment for developing intelligent headlight systems," in Proc. IEEE Intell. Veh. Symp., San Diego, CA, USA, Jun. 2010, pp. 225-231.

[6] United Nations Economic Commission for Europe, Type-Approval Requirements for the General Safety of Motor Vehicles, Add. 37, Rev. 2, Amend. 2, Rear Fog Lamps, 2007.

[7] E. Dumont and V. Cavallo, "Extended photometric model of fog effects on road vision," Transp. Res. Rec., no. 1862, pp. 77-81, Jan. 2004.

[8] N. Hautière, J.-P. Tarel, J. Lavenant, and D. Aubert, "Automatic fog detection and estimation of visibility distance through use of an onboard camera," Mach. Vis. Appl., vol. 17, no. 1, pp. 8-20, Apr. 2006.

[9] S. Bronte, L. Bergasa, and P. Alcantarilla, "Fog detection system based on computer vision techniques," in Proc. IEEE Conf. Intell. Transp. Syst., Saint-Louis, MO, USA, Oct. 2009, pp. 1-6.

[10] S. Lenor, B. Jähne, S. Weber, and U. Stopper, "An improved model for estimating the meteorological visibility from a road surface luminance curve," Lect. Notes Comput. Sci., vol. 8412, pp. 184-193, 2013.

[11] N. Hautière, J.-P. Tarel, and D. Aubert, "Mitigation of visibility loss for advanced camera based driver assistances," IEEE Trans. Intell. Transp. Syst., vol. 11, no. 2, pp. 474-484, Jun. 2010.

[12] J.-P. Tarel et al., "Vision enhancement in homogeneous and heterogeneous fog," IEEE Intell. Transp. Syst. Mag., vol. 4, no. 2, pp. 6-20, 2012.

[13] L. Caraffa and J.-P. Tarel, "Markov random field model for single image defogging," in Proc. IEEE IV, Gold Coast, QLD, Australia, Jun. 2013, pp. 994-999.

[14] R. Gallen, A. Cord, N. Hautière, and D. Aubert, "Towards night fog detection through use of in-vehicle multipurpose cameras," in Proc. IEEE Intell. Veh. Symp., Jun. 2011, pp. 399-404.

[15] M. Pavlic, G. Rigoll, and S. Ilic, "Classification of images in fog and fogfree scenes for use in vehicles," in Proc. IEEE IV Jun. 2013, pp. 481-486.
[16] J. Leleve, A. Bensrhair, and J. Rebut, "Method for detecting night fog and system implementing said method," EP Patent 1715456 B 1, Aug. 27, 2008.

[17] N. Kawasaki, T. Miyahara, and Y. Tamatsu, "Visibility condition determining device for vehicle," Patent 20080007429 A1, Jan. 10, 2008.

[18] S. G. Narasimhan and S. K. Nayar, "Vision and the atmosphere," Int. J. Comput. Vis., vol. 48, no. 3, pp. 233-254, Jul. 2002.

[19] S. Narasimhan and S. Nayar, "Shedding light on the weather," in Proc. IEEE Conf. Comput. Vis. Pattern Recog., 2003, pp. 665-672.

[20] S. Metari and F. Deschênes, "A new convolution kernel for atmospheric point spread function applied to computer vision," in Proc. IEEE Int. Conf. Comput. Vis., Rio de Janeiro, Brazil, Oct. 2007, pp. 1-8.

[21] R. Gallen, N. Hautière, and E. Dumont, "Static estimation of the meteorological visibility distance in night fog with imagery," IEICE Trans. Inf. Syst., vol. E93-D, no. 7, pp. 1780-1787, Jul. 2010.

[22] E. Dumont, R. Brémond, and N. Hautière, "Night-time visibility as a function of headlamp beam pattern and pavement reflection properties," in Proc. Int. Congr. VISION, Versailles, France, 2008, pp. 1-7.

[23] H. R. Blackwell, "Contrast threshold of the human eye," J. Opt. Soc. Am., vol. 36, no. 11, pp. 624-632, Nov. 1946.

[24] "A Unified Framework of Methods for Evaluating Visual Performance Aspects of Lighting," Paris, France, Tech. Rep. 19-2, 1972.

[25] W. Adrian, "Visibility of targets: A model for calculation," Lighting Res. Technol., vol. 21, no. 4, pp. 181-188, Dec. 1989.

[26] W. Adrian, "Visibility levels under night-time driving conditions," J. Illuminating Eng. Soc., vol. 16, no. 2, pp. 3-12, 1987.

[27] P. Olson, T. Aoki, D. Battle, and M. Flannagan, "Development of a Headlight System Performance Evaluation Tool," UMTRI, Ann Arbor, MI, USA, Tech. Rep. UMTRI-90-41, Oct. 1990.

[28] "Road Surfaces and Lighting," Vienna, Austria, Tech. Rep. 66, Jan. 1984.

[29] E. Farber and V. Bhise, "Development of a Headlight Evaluation Model," Washington, DC, USA, TRB Special Report 156, 1975.

[30] E. Dumont, N. Hautière, and R. Gallen, "A semi-analytic model of fog effects on vision," in Atmospheric Turbulence, Meteorological Modeling and Aerodynamics. Commack, NY, USA: Nova, 2010, pp. 635-670.

[31] E. Dumont, "Semi-monte-carlo light tracing for the study of road visibility in fog," in Monte Carlo and Quasi-Monte Carlo Methods 1998. Berlin, Germany: Springer-Verlag, 1999, pp. 177-187.

[32] B. Schoettle, M. Sivak, and M. Flannagan, "Highbeam and Low-Beam Headlighting Patterns in the US and Europe at the Turn of the Millennium," UMTRI, Ann Arbor, MI, USA, Tech. Rep. 19, May 2001.

[33] M. Colomb et al., "An innovative artificial fog production device improved in the European project FOG," Atmos. Res., vol. 87, no. 3/4, pp. 242-251, Mar. 2008.

[34] N. Otsu, "A threshold selection method from gray-level histograms," IEEE Trans. Syst., Man, Cybern., vol. 9, no. 1, pp. 62-66, Jan. 1979.

[35] R. Gallen, A. Cord, N. Hautière, and D. Aubert, "Method and device for detecting fog at night," Int. Patent No. WO 2012042171 A2, Versailles, France, Apr. 2012.

[36] R. Gallen, E. Dumont, and N. Hautière, "A conventional approach to nighttime visibility in adverse weather conditions," in Proc. ISAL, Darmstadt, Germany, Sep. 26-28, 2011.

[37] N. Hautière, E. Bigorgne, J. Bossu, and D. Aubert, "Meteorological conditions processing for vision-based traffic monitoring," in Proc. IEEE Int. Workshop VS, Marseille, France, Oct. 2008, pp. 1-8.

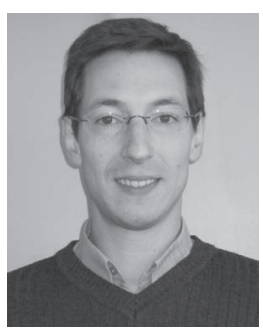

Romain Gallen received the M.S. degree in computer vision for industrial applications and the Ph.D. degree from Université Pierre et Marie Curie, Paris, France, in 2005 and 2010, respectively.

From 2005 to 2007 he was an Engineer with the Laboratory on Interaction Between Vehicle Infrastructure and Driver (LIVIC), which is a research unit in the Department of Components and Systems, The French Institute of Science and Technology for Transport, Development and Networks (IFSTTAR), Versailles, France. From 2007 to 2010, during his Ph.D. studies, he focused on visibility risk assessment in adverse weather conditions using computer vision with both LIVIC and the Laboratory for Road Operation, Perception, Simulations and Simulators (LEPSIS), Department of Components and Systems, IFSTTAR. Since 2011 he has been the Head of the Information Processing Unit, Direction Technique Eau, mer et fleuves (DTecEMF), Centre d'Etudes et d'Expertises sur les Risques, l'Environnement, la Mobilité et l'Aménagement (CEREMA), Plouzané, France, where he deals with maritime transport safety. 


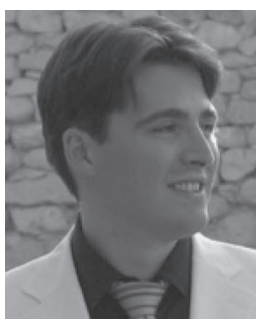

Aurélien Cord received the Ph.D. degree in image processing for planetary surfaces from Toulouse University, Toulouse, France, in 2003.

In 2004 he was working on content-based image retrieval with Heuristique et Diagnostic des Systémes Complexes (Heudiasyc), Compiègne University, Compiègne, France. In 2005 he was working on the photometrical and spectral analysis of images from the Mars Express European mission with the European Space Agency. From 2006 to 2007 he was working on the automatic characterization of textured images for defect detection on steel plate images with Centre de Morphologie Mathématique, École Nationale Supérieure des Mines de Paris (Mines ParisTech), Paris, France. Since 2008 he has been a Researcher with the Laboratory on Interaction Between Vehicle Infrastructure and Driver (LIVIC), Department of Components and Systems, The French Institute of Science and Technology for Transport, Development and Networks (IFSTTAR), Versailles, France, where he works on computer vision applied to intelligent transportation systems. His research interests include the analysis and interpretation of images and videos from onboard cameras.

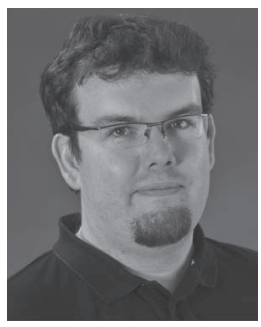

Nicolas Hautière (M'10) received the M.S. degree in civil engineering from National School of State Public Works (ENTPE), Vaulx-en-Velin, France, in 2002; the M.S. and Ph.D. degrees in computer vision from Université Jean Monnet, Saint-Étienne, France, in 2002 and 2005, respectively; the Habilitation to manage research (HDR) from Université Paris-Est, Champs sur Marne, France, in 2011; and the Specialized Master's degree in political science and sustainable development from École des Ponts ParisTech, Champs-sur-Marne, France, in 2013.

Since September 2013 he has been a Project Director with the Department of Components and Systems, The French Institute of Science and Technology for Transport, Development and Networks (IFSTTAR), Marne la Vallée, France. His research interests include the modeling of meteorological phenomena reducing highway visibility, the detection of visibility conditions, and the estimation of the visibility range. Since 2010, he has been a Member of the IEEE and of the IEEE Intelligent Transportation Systems Society

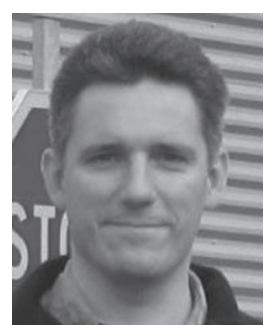

Éric Dumont received the M.S. degree in civil engineering from National School of State Public Works (ENTPE), Lyon, France, in 1992; the M.S. degree in image processing from Université Jean Monnet, Saint-Étienne, France, in 1992; and the $\mathrm{Ph} . \mathrm{D}$. degree in mathematics and computer science from the René Descartes University, Paris, France, in 2002.

$\mathrm{He}$ is a Senior Research Engineer with and the Deputy Director of the Laboratory for Road Operation, Perception, Simulations and Simulators (LEPSIS), Department of Components and Systems, The French Institute of Science and Technology for Transport, Development and Networks (IFSTTAR), Marne la Vallée, France. His research interests include the synthesis, acquisition, and analysis of digital images for the assessment of road visibility in various lighting and weather conditions.

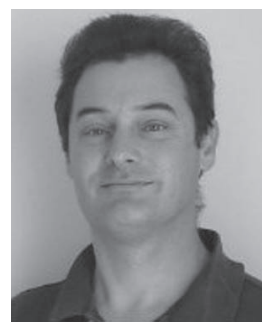

Didier Aubert received the M.S. and Ph.D. degrees from National Polytechnical Institute of Grenoble (INPG), Grenoble, France, in 1985 and 1989, respectively.

From 1989 to 1990 he was a Research Scientist on the development of an automatic road following system with the Navigation Laboratory (NAVLAB), Carnegie Mellon University, Pittsburgh, PA, USA. From 1990 to 1994 he was with the research department of a private company, Industrie et Technologie de la Machine Intelligente. During this period, he was a Project Leader of several projects dealing with computer vision. In 1995 he joined the French National Institute for Transportation and Safety Research (INRETS), which later became The French Institute of Science and Technology for Transport, Development and Networks (IFSTTAR), Versailles, France. He is currently a Senior Researcher with and the Director of the Laboratory for Road Operation, Perception, Simulations and Simulators (LEPSIS), Department of Components and Systems, IFSTTAR. He works on road traffic monitoring, crowd monitoring, automated highway systems, and driving assistance systems. $\mathrm{He}$ is an Image Processing Expert for several companies and teaches in several universities [Université Pierre et Marie Curie (Paris VI), Paris, France; Université Paris-Sud (Paris XI), Paris, France; Université Paris-Est Créteil Val-de-Marne (Paris XII), Paris, France; and Evry, Versailles, France) and engineering schools (École des Ponts ParisTech, Champs-sur-Marne, France; École Nationale Supérieure des Télécommunications ParisTech, Paris, France; and École Nationale Supérieure des Mines de Paris (Mines ParisTech), Paris, France]. 